\title{
Pulmonary Necrobiotic Nodules in Crohn's Disease: A Rare Extra-intestinal Manifestation
}

\author{
Karim El-Kersh MD, Mostafa Fraig MD, Rodrigo Cavallazzi MD, \\ Mohamed Saad MD, and Rafael L Perez MD
}

\begin{abstract}
Pulmonary necrobiotic nodules represent a rare extra-intestinal manifestation of Crohn's disease. Histologically, they are composed of sterile aggregates of inflammatory cells with necrosis. The differential diagnosis is broad, and exclusion of infectious etiologies is mandatory before starting immunosuppressive therapy. Here, we present the fifth reported case of pulmonary necrobiotic nodules in Crohn's disease. Our patient had new-onset Crohn's disease associated with both cavitating and non-cavitating lung nodules that were confirmed to be necrobiotic nodules by biopsy. The patient was started on mesalamine and prednisone, with subsequent improvement of his gastrointestinal symptoms and regression of the necrobiotic nodules. Key words: pulmonary necrobiotic nodules; Crohn's disease; video-assisted thoracoscopy. [Respir Care 2014;59(12):e190-e192. (C) 2014 Daedalus Enterprises]
\end{abstract}

\section{Introduction}

Crohn's disease is an inflammatory bowel disease (IBD) of unknown etiology that can affect any part of the gastrointestinal tract. Although the extra-intestinal manifestations of IBD most commonly affect musculoskeletal, ocular, and mucocutaneous systems, they can also involve the lungs. In 2000, Faller et $\mathrm{al}^{1}$ described the first case of pulmonary necrobiotic nodules in association with Crohn's disease. Only a few similar cases have subsequently been reported. ${ }^{2-4}$ Here, we present the fifth reported case of pulmonary necrobiotic nodules in association with Crohn's disease, with a brief review of the relevant literature.

Drs El-Kersh, Cavallazzi, Saad, and Perez are affiliated with the Department of Pulmonary, Critical Care, and Sleep Disorders Medicine, and Dr Fraig is affiliated with the Department of Pathology, University of Louisville, Louisville, Kentucky.

The authors have disclosed no conflicts of interest.

Drs El-Kersh, Perez, and Fraig presented a version of this paper at Chest 2013, held October 26-31, 2013, in Chicago, Illinois.

Correspondence: Karim El-Kersh MD, Department of Pulmonary, Critical Care, and Sleep Disorders Medicine, Ambulatory Care Building, 550 S Jackson Street, Louisville, KY 40202. E-mail: kaelke01 @ louisville.edu.

DOI: $10.4187 /$ respcare. 03176

\section{Case Report}

A 22-y-old male with no significant past medical history presented with intermittent abdominal pain, fever, and bloody bowel movements for 1 month. He was not receiving any medical therapy, but he used acetaminophen for his fever. His abdominal pain was diffuse, non-radiating, and associated with frequent bowel movements with bright red blood. The patient was a non-smoker with no history of drug abuse. There was no history of recent travels or recent dental extractions. He denied neck pain or sinus symptoms. His past surgical history included an uncomplicated appendectomy $5 \mathrm{y}$ ago. The patient denied joint pain, chest pain, cough, or shortness of breath.

On presentation, the patient was afebrile with stable vitals. A complete physical examination was unremarkable apart from a diffuse abdominal tenderness on deep palpation with no rebound. There was no hepatosplenomegaly, and the chest was clear to auscultation bilaterally.

The complete blood cell count was normal apart from microcytic anemia with a hemoglobin level of $10.6 \mathrm{~g} / \mathrm{dL}$ (normal range: $13.7-17.5 \mathrm{~g} / \mathrm{dL}$ ). A complete metabolic panel, including electrolytes, renal and liver function testing, amylase, and lipase, was within normal limits. C-reactive protein was elevated at $9.60 \mathrm{mg} / \mathrm{dL}$ (normal range: $0-0.49 \mathrm{mg} / \mathrm{dL}$ ). Blood cultures were negative. Abdominal computed tomography showed evidence of colitis. 


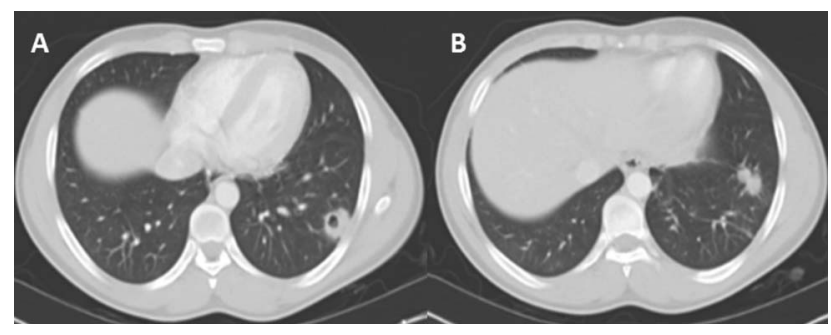

Fig. 1. Chest computed tomography shows a left lower lobe cavitary lesion measuring $\sim 3 \mathrm{~cm}$ in the long axis (A) and 2 noncavitary lesions (B).

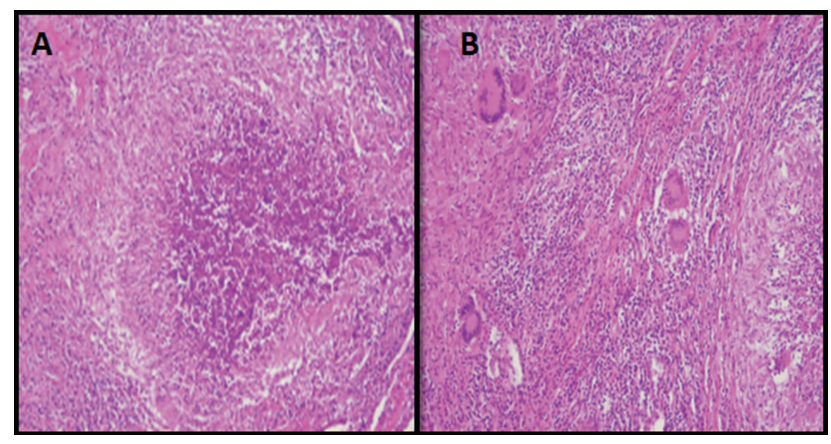

Fig. 2. A: Necrobiotic nodule with central necrosis and palisading histiocytes at the periphery surrounded by an area of chronic inflammation and fibrosis. Special stains for fungal and mycobacterial microorganisms (not shown) were negative (hematoxylin and eosin stain, magnification $\times 10$ ). B: Necrobiotic nodule with central necrosis and multinucleated giant cells at the periphery similar to the granulomatous reaction seen in the gastrointestinal tract of Crohn's disease patients (hematoxylin and eosin stain, magnification $\times 10$ ).

Cavitary and non-cavitary lesions were noted in the left lower lobe (Fig. 1). The patient underwent colonoscopy, with multiple colonic and ileal biopsies that were consistent with active Crohn's disease. QuantiFERON-TB (Cellestis Limited, Carnegie, Victoria, Australia) Gold testing, human immunodeficiency virus testing, sputum for acid-fast bacilli smear and culture, serum fungal serologies, rheumatoid factor, and anti-neutrophil cytoplasmic antibody testing (including anti-proteinase 3 and anti-myeloperoxidase) were negative. The serum angiotensinconverting enzyme level was normal. A transthoracic needle biopsy was not diagnostic. The patient underwent a left lower lobe wedge biopsy by video-assisted thoracoscopy, which confirmed the diagnosis of pulmonary necrobiotic nodules (Fig. 2). He was started on mesalamine and prednisone, with subsequent improvement of his gastrointestinal symptoms and regression of the necrobiotic nodules.

\section{Discussion}

The incidence of extra-intestinal manifestations in IBD ranges from 21 to $41 \%$, but the true incidence of pulmo-
Table 1. Respiratory Involvement in Crohn's Disease

\begin{tabular}{lc}
\hline \hline Anatomic Location & \multicolumn{1}{c}{ Involvement } \\
\hline Airways & $\begin{array}{c}\text { Large and small airway inflammation causing } \\
\text { bronchitis, tracheobronchial stenosis, } \\
\text { bronchiectasis, enteric pulmonary fistula } \\
\text { formation, or bronchiolitis obliterans }\end{array}$ \\
Lung parenchyma & $\begin{array}{c}\text { Interstitial lung diseases, including organizing } \\
\text { pneumonia, nonspecific interstitial pneumonitis, } \\
\text { and lung infiltrates with peripheral eosinophilia. } \\
\text { Necrobiotic lung nodules (rare) }\end{array}$ \\
Pulmonary artery & $\begin{array}{c}\text { Pulmonary venous thromboembolism } \\
\text { Serous membranes }\end{array}$ \\
& $\begin{array}{c}\text { Serositis that can result in pleuropericarditis and } \\
\text { pleural effusions (rare) }\end{array}$ \\
\hline
\end{tabular}

nary involvement remains unknown. ${ }^{5}$ The pathogenesis of lung involvement associated with IBD is uncertain, but the common embryologic origin of the lungs and gastrointestinal tract may theoretically play a role through shared antigenicity. $.5,6$

Pulmonary involvement in IBD can represent a diagnostic challenge especially if it occurs in a patient who is maintained on steroid-sparing agents for IBD, such as sulfasalazine, mesalamine, azathioprine, 6-mercaptopurine, methotrexate, or biologic therapeutic agents. A broad spectrum of pulmonary involvement (mainly interstitial) has been described in association with such treatments. ${ }^{6}$ In this situation, it is important to distinguish between pulmonary involvement as an extra-intestinal manifestation of IBD and drug-induced pulmonary complications. Bronchoalveolar lavage characteristics can be helpful in differentiation, with a lymphocytic bronchoalveolar lavage found in Crohn's disease-related interstitial lung diseases versus an eosinophilic bronchoalveolar lavage in drug-induced pulmonary interstitial complications.

As opposed to ulcerative colitis, respiratory involvement occurs less commonly in patients with Crohn's disease and can be grouped into 4 categories, with a rare occurrence of serositis and necrobiotic lung nodules in association with Crohn's disease (Table 1). ${ }^{7,8}$

Necrobiotic lung nodules are sterile nodules that can cavitate. Histologically, they are composed of sterile aggregates of inflammatory cells with necrosis and have a high degree of resemblance to pyoderma gangrenosum in cases of IBD and to subcutaneous rheumatoid nodules in cases of rheumatoid arthritis. ${ }^{7}$

Originally, necrobiotic lung nodules were described in patients with rheumatoid arthritis. These nodules, in association with pneumoconiosis, constitute Caplan syndrome. In rheumatoid arthritis, necrobiotic lung nodules usually have pleural or subpleural locations. They can be complicated by hemoptysis or rupture into the pleural space, resulting in pneumothorax, hydropneumothorax, pyopneumothorax, and bronchopleural fistulas. ${ }^{9}$ Subsequently, 
necrobiotic lung nodules were described with ulcerative colitis, but rarely with Crohn's disease. ${ }^{1-4,7}$

To our knowledge, only 4 cases of pulmonary necrobiotic nodules in association with Crohn's disease have been reported. Two patients were males, and 2 were females. The ages at presentation ranged from 22 to $57 \mathrm{y}$. The presenting symptoms varied from cough and dyspnea to chest pain. Intestinal disease was active in 2 of the reported cases at the time of diagnosis of necrobiotic lung nodules. On radiological examination, only 2 of the reported patients had evidence of cavitation of their lung nodules. ${ }^{4}$

Cavitating necrobiotic lung nodules have a radiographic appearance similar to the cavitating nodules of malignancy, autoimmune diseases such as granulomatosis with polyangiitis (formerly Wegener granulomatosis), septic pulmonary emboli, and other infectious etiologies, especially fungal infections and tuberculosis. Exclusion of these infectious etiologies is prudent to avoid dissemination of infection upon starting immunosuppressive therapy. ${ }^{10}$ Nodular pulmonary amyloidosis associated with Crohn's disease and focal non-granulomatous lymphoid infiltration are other rare causes of lung nodules in Crohn's disease that can mimic necrobiotic lung nodules. ${ }^{11,12}$

The small number of reported cases of necrobiotic nodules complicating Crohn's disease do not allow strong conclusions regarding therapy. Of the 4 reported patients, 3 responded to oral prednisone with radiological resolution, and one patient who was maintained on mesalamine at the time of diagnosis had complete radiological resolution with no additional therapy.,2,4

In conclusion, despite their rarity, pulmonary necrobiotic nodules should be considered in the differential diag- nosis of lung nodules in patients with Crohn's disease. Exclusion of an infectious etiology is mandatory prior to starting treatment.

\section{REFERENCES}

1. Faller M, Gasser B, Massard G, Pauli G, Quoix E. Pulmonary migratory infiltrates and pachypleuritis in a patient with Crohn's's disease. Respiration 2000;67(4):459-463.

2. Sanjeevi A, Roy HK. Necrobiotic nodules: a rare pulmonary manifestation of Crohn's's disease. Am J Gastroenterol 2003;98(4):941943.

3. Freeman HJ, Davis JE, Prest ME, Lawson EJ. Granulomatous bronchiolitis with necrobiotic pulmonary nodules in Crohn's's disease. Can J Gastroenterol 2004;18(11):687-690.

4. Warwick G, Leecy T, Silverstone E, Rainer S, Feller R, Yates DH. Pulmonary necrobiotic nodules: a rare extraintestinal manifestation of Crohn's's disease. Eur Respir Rev 2009;18(111):47-50.

5. Storch I, Sachar D, Katz S. Pulmonary manifestations of inflammatory bowel disease. Inflamm Bowel Dis 2003;9(2):104-115.

6. Casella G, Villanacci V, Di Bella C, Antonelli E, Baldini V, Bassotti G. Pulmonary diseases associated with inflammatory bowel diseases. J Crohn'ss Colitis 2010;4(4):384-389.

7. Camus $P$, Colby TV. The lung in inflammatory bowel disease. Eur Respir J 2000;15(1):5-10.

8. Murthy SK, Nguyen GC. Venous thromboembolism in inflammatory bowel disease: an epidemiological review. Am J Gastroenterol 2011; 106(4):713-718.

9. Walters MN, Ojeda VJ. Pleuropulmonary necrobiotic rheumatoid nodules. A review and clinicopathological study of six patients. Med J Aust 1986;144(12):648-651.

10. Schleiermacher D, Hoffmann JC. Pulmonary abnormalities in inflammatory bowel disease. J Crohn'ss Colitis 2007;1(2):61-69.

11. Golpe R, Mateos A, Pérez-Valcárcel J, Lapeña JA, García-Figueiras R, Blanco J. Multiple pulmonary nodules in a patient with Crohn's's disease. Respiration 2003;70(3):306-309.

12. Beer TW, Edwards CW. Pulmonary nodules due to reactive systemic amyloidosis (AA) in Crohn's's disease. Thorax 1993;48(12):12871288. 